\title{
Commissioning of the ATLAS Pixel Detector and operational experience with first data
}

\author{
S. Montesano ${ }^{\mathrm{a} *}$, on behalf of the ATLAS Collaboration \\ ${ }^{a}$ INFN Sezione di Milano, Via Celoria 16, 20133 Milano, Italy and \\ European Organization for Nuclear Research, CERN, CH-1211, Genève 23, Switzerland
}

The Pixel Detector is the innermost component of the ATLAS tracking system. It was commissioned since 2008 with cosmic ray data and more recently with $p p$ collisions at $\sqrt{s}=900 \mathrm{GeV}$ and $\sqrt{s}=7$ TeV. The read-out electronics was tuned, detection efficiency was measured and noise suppression was implemented. The study of cluster properties allowed to improve spatial resolution and to identify particles by measuring their energy loss.

\section{Introduction}

ATLAS [1] is a general purpose particle physics experiment at the Large Hadron Collider at CERN. The Pixel Detector [2] is the innermost component of its tracker: it is made of three barrel layers (with radius $50.5 \mathrm{~mm}, 88.5 \mathrm{~mm}$ and $122.5 \mathrm{~mm}$ ) and two end-caps, each one constituted of three disks. The basic components of the detector are the modules, that are identical for barrel and disks. In the barrel they are arranged in overlapping staves of 13 modules each, while in the disks they form 6-modules sectors. Each module contains 47,232 pixels with a total of more than $80 \times 10^{6}$ pixels over a surface of 1.7 $\mathrm{m}^{2}$.

The detector structure is made of low-mass carbon fiber and integrates the cooling system, resulting in a total contribution to $X_{0}$ of about $3 \%$ per layer. A radiation dose of $\sim 500 \mathrm{kGy}(\mathrm{Si})$ is expected during detector life time. The read-out clock is given by the LHC 25 ns bunch crossing (BC). A resolution of $10 \mu \mathrm{m}$ in the azimuthal direction and $115 \mu \mathrm{m}$ along the beam direction are required, imposing an alignment precision better than $10 \mu \mathrm{m}$.

\section{The Pixel Detector module}

Each module is composed by a sensor, 16 frontend (FE) chips and a flex hybrid containing control and transmission electronics.

\footnotetext{
*simone.montesano@cern.ch
}

The FE chips are bump-bonded to the pixels using solder or indium bonds with a $50 \mu \mathrm{m}$ pitch. Each chip contains $18 \times 160$ cells, realized with $250 \mathrm{~nm}$ CMOS technology. The FEs provide charge measurement using the time-overthreshold of the output signal (Sec. 4).

The sensor is made of $n^{+}$-in- $n$ silicon with typical pixels size of $50 \times 400 \mu \mathrm{m}^{2}$ (short side is along the $\mathrm{R} \phi$ direction). Some pixels with modified geometries (long and ganged pixels) are present near to the borders of the FE. The measured sensor thickness is $256 \pm 3 \mu \mathrm{m}$ [2].

A flexible kapton circuit, wire-bonded to the FE and glued on the reverse of the sensor, provides a bias voltage of $150 \mathrm{~V}$. It contains the Module Controller Chip (MCC) that controls the FE and has basic event reconstruction capability.

\section{Data taking operations}

The Pixel Detector was installed in June 2007. The commissioning with cosmic muon data started in September 2008. More than 600,000 tracks were collected. Since November 2009, the detector is involved in collision data taking: more than $500 \times 10^{6}$ candidates have been recorded, at center-of-mass energies of $900 \mathrm{GeV}$ and, starting from April 2010, $7 \mathrm{TeV}$. Special procedures exist to switch the detector on and off, in order to ensure that high voltage is provided only when LHC beam is stable.

The intrinsic efficiency was measured by extrapolating cosmic ray tracks through the detec- 


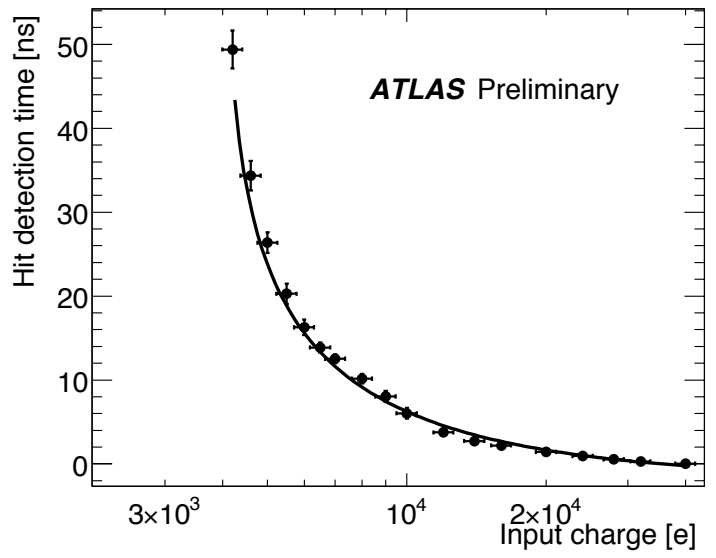

Figure 1. Hit detection time as a function of the injected charge. A MIP corresponds to 20,000 e, with a detection time close to $2 \mathrm{~ns}$. tor and counting the numbers of measurements pected but is not found. The measured efficiency was $(99.974 \pm 0.004$ (stat.) \pm 0.003 (syst.))\% for the barrel part, if non-operational modules are excluded [3]. Irreversible failures involve 46 modules that correspond to $2.64 \%$ of the detector.

\section{Calibrations of the read-out electronics}

The read-out FE contains a charge amplifier with constant-current feedback that ensures high stability and fast signal shaping through a linear decay of the shaped signal. The charge collected by each pixel is estimated by the time during which the signal is higher than a configurable threshold. The threshold is set for each module and can be trimmed at pixel level, by using a 7bit DAC. The feedback current itself can be tuned with a 3-bit DAC for each read-out channel.

A threshold of $4000 e$ was initially used. After tuning, the distribution of threshold values showed high uniformity, with an RMS of $40 e$. The typical electronic noise was $200 e$ for normal pixels, giving a very favourable threshold over noise ratio. For this reason, the threshold has been lowered to $3500 e$ for 2010 data-taking.

The feedback current is configured in order to have a $30 \mathrm{BC}$ time-over-threshold (ToT) for a 20,000 e minimum ionizing particle (MIP). The on the track and holes where a hit would be ex- resolution on ToT measurement is described by the formula $\sigma_{\mathrm{ToT}}=p_{1}+p_{2} \times$ ToT, where $p_{1}$ and $p_{2}$ are parameters fitted from data. For a MIP, the resolution is about $1 \mathrm{BC}(660 \mathrm{e})$, higher than the electronic noise.

Due to the delay with which it exceeds the threshold, a hit can be associate to the correct $\mathrm{BC}$ or to the following one. This time-walk effect is studied by injecting known charges into the read-out electronics and measuring the detection delay (Fig. 1). The in-time threshold is defined as the minimal signal needed to have correct hit-BC assignment. This has been measured to be $4870 e$ (with 158 e dispersion) for the $3500 e$ threshold.

During cosmic ray data taking, a read-out window of $5 \mathrm{BC}$ was used. After assessing a better module synchronization, this was reduced to $4 \mathrm{BC}$ in 2010, getting a read-out efficiency of $99.95 \%$ for hits associated to tracks. Hits out of the main $\mathrm{BC}$ are mainly located at the edge of the modules, where some charge can be lost, or in the ganged pixel region, where each read-out channel serves two different pixels.

\section{Noise masking}

The noise rate is dominated by few hundred pixels. For this reason, noise is reduced either by excluding pixels from read-out (online mask) or by not using them in data reconstruction (offline mask). The online mask is provided for each readout configuration, while the offline mask is computed by using a calibration loop. During each data-taking run, a calibration stream is provided by triggering empty bunch crossing: pixels are considered noisy if they have an occupancy larger than $10^{-5}$ hits per BC. The reconstruction of the bulk of data is delayed by 36 hours and is performed masking the noisy pixels just identified: the noise measured is lower than $10^{-9}$ hits per pixel per BC.

\section{Lorentz angle measurement}

ATLAS uses a $2 \mathrm{~T}$ solenoidal magnetic field to measure the momentum of particles. The field causes the charge carriers inside the Pixel Detector sensor to drift towards the electrodes forming 


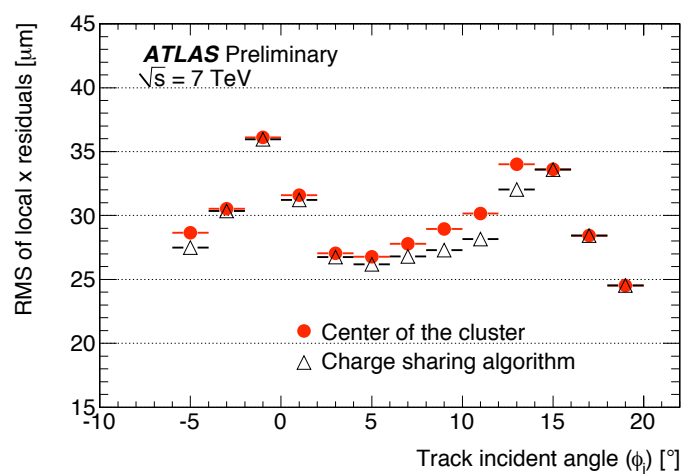

Figure 2. RMS of residual distribution for cluster local y position with respect to track extrapolation. The distribution is plotted as a function of the track incident angle.

an angle with respect to the normal to the module surface, the Lorentz angle. When estimating the particle crossing point, this angle should be subtracted from the incident angle of particles [4].

The distribution of the cluster size as a function of the particle incident angle is fit to measure the Lorentz angle, that corresponds to the minimum of this distribution. A full study of the systematic uncertainties was performed on cosmic ray data, giving a value of $11.77^{\circ} \pm 0.03^{\circ} \pm_{0.23^{\circ}}^{0.13^{\circ}}$, while the theory predicts $12.9^{\circ} \pm 1.55^{\circ}[3]$. On collision data, the measurement is less accurate, due to the smaller range of particle incident angles. The mean temperature of the detector is lower, giving a higher measured value of $12.1^{\circ} \pm 0.09^{\circ}$ for a threshold of $3500 \mathrm{e}$.

\section{Charge sharing and spatial resolution}

The spatial resolution depends on read-out tuning, on cluster size and on particle incident angle. It is improved by summing to the geometrical center of the cluster a correction term proportional to charge sharing. The charge sharing variable is defined as the ratio between the charge collected in the first pixel of the cluster and the total charge collected in the outermost pixels. The weight of the correction is fit from data for different cluster sizes and ranges of incident angle [5].

Fig. 2 shows the RMS of residual distribution with respect to the track extrapolation for the lo- cal $x$ position of clusters, as a function of the track incident angle. The residual distribution width is determined both by extrapolation uncertainty and by detector resolution. The improvement due to charge sharing correction is clearly visible.

\section{Energy loss and particle identification}

The specific energy loss $(\mathrm{d} E / \mathrm{d} x)$ associated to particles is measured from the charge collected in the clusters, after a selection that excludes regions where charge collection is poor. The track $\mathrm{d} E / \mathrm{d} x$ is defined as the truncated average of the individual cluster energies, by removing the highest charge, in order to reduce the Landau tails.

After plotting the dependence of $\mathrm{d} E / \mathrm{d} x$ on track momentum and charge, separated bands for pions, kaons and (anti-)protons are identified, in particular in the low momentum region.

\section{Conclusions}

The Pixel Detector was commissioned with cosmic ray data in 2008 and 2009 and with LHC collision data in 2009 and 2010. The commissioning activity allowed to uniformly tune the readout electronics response to injected charge and to improve the synchronization of all the modules composing the device. The hit-track association efficiency and the noise rate were measured and an algorithm was developed in order to identify and exclude noisy pixels from track reconstruction. The study of cluster properties allowed to improve the spatial resolution by using information about charge sharing among hit pixels and by precisely measuring the Lorentz angle. Furthermore particle identification was implemented, by exploiting information about particle specific energy loss in the silicon sensors.

\section{REFERENCES}

1. ATLAS collab.. JIST, 3(08):S08003, 2008.

2. G. Aad et al. JIST, 3(07):P07007, 2008.

3. ATLAS collab.. The ATLAS Inner Detector commissioning and calibration, arXiv:1004.5293.

4. I. Gorelov et al. NIMA, 481:204-221, 2002.

5. S. Montesano. CERN-THESIS-2010-018 